\title{
Toward A Full Implementation of the Operation and Support Subsystem (OSS) in IMS Networks
}

\author{
Nguyen Tai Hung ${ }^{1}$, Nguyen Huu Thanh ${ }^{1}$, Julius Mueller ${ }^{2}$ \\ ${ }^{1}$ Hanoi University of Science and Technology, Hanoi, Vietnam \\ 2 Technical University of Berlin, Berlin, Germany
}

Correspondence: Nguyen Tai Hung, hung.nguyentai@hust.edu.vn

Manuscript communication: received 22 December 2013, accepted 19 May 2014

\begin{abstract}
Recently, mobile operators all over the world are facing big challenges on the decreasing of the revenues from its traditional killer-applications like SMS/MMS and even voice services. The challenges come from a new trend of services called OTT (Over The Top). These OTT services provide the mobile users with flexible, convenient and more importantly free means to do their daily multimedia communication needs and seamlessly without any border so that operators couldn't be just simply block it, but in opposite, need to find solutions to compete against it. Because of that, nowadays, more and more operators choose to deploy the standard RCS (Rich Communication Suite). RCS is a set of rich features communication services developed on top of IMS framework. Although there have been tremendous efforts to develop the architecture and protocols for IMS, which is a key technology of the NGN, it is far from being widely deployed. There are many reasons for that unpopularity (of IMS commercial deployment), namely, the lacking of clear business model for IMS-based services, no big difference between IMS-based services and SOA (Service Oriented Architecture) services, lack of the true demands for features that IMS based services have, etc... However, one of the most important reason that till now not many researchers have pointed out and addressed it, is about the OSS (Operation and Support Subsystem) functions. The lacking of OSS clearly defined functions and interfaces make the difficulties for operators and even equipment manufacturers to collect the pay back for any big scale IMS deployment for their networks. This paper presents our proven that IMS can provide the killer applications with blending features like mash-up and readily equipped with the intelligent charging and policy control functions. That may partly help to speed up the deployment process of IMS in real networking environment.
\end{abstract}

Keywords- IMS, LES, Online Charging, Offline Charging, Ro/Rf, Blended Services, Combined Services, GARC, Policy Control.

\section{INTRODUCTION}

With the rapid growth of the network infrastructure and technologies such as SOA/Web 2.0 [1] (Service Oriented Architecture) and IMS [2] (IP Multimedia Subsystem), end-users are looking for richer communication services to suite their lifestyles and needs. Service Providers are facing multiple challenges of adapting their network infrastructure to leverage the existing silo services to provide composite services which can span across multiple networks. In the current scenario telecom service providers offer their customers the silo services in the form of service packages. Service providers can enrich their service-offering by dynamically blending the individual silo telecom services and Web 2.0 services to create new breed of richer telecom services. Such services are called blended services which can be either static or dynamic in nature. The static blended service is invocation of service enablers in a predefined order. Whereas dynamic blended services represent a complex activity specified by composing multiple service enablers spanning across diversified networks based on the user contextual data and operator defined rules associated with in a single session. The future IMS based networks will be dynamically delivering a blend of one or more such services simultaneously to the end-users based on their preferences and context. 3GPP (Third Generation Partnership Project) proposed SCIM [3] (Service Capability Interaction Manager) as the network element in the IMS architecture which is responsible for delivery of the blended services. Blended services might involve invocation of both SIP as well as Non-SIP based application servers. Charging of such dynamic blended services is important and is a real challenge for operators or service providers, especially with policy-based charging. IMS networks can be successful if they are proven to be revenue generating. IMS networks facilitate both offline and online charging of multimedia services. Offline charging is a process where the charging data doesn't affect the service rendered in real time. It means that the subscriber is charged for his service/network usage after the service delivery. In contrast, online charging is a process where the charging data affects the service rendered in real time. The subscriber's service or network usage is monitored and controlled by a centralized node called as Online Charging System (OCS). The subscriber's account balance is debited in real time based on his usage.

The Location Enabled Services (LES) is one of the blended services that require dynamic rules for invocation of service features on both SIP and Non- 
SIP (Parlay-X/Web2.0) application platforms during single user session. This paper presents our proposal of a comprehensive OSS framework and a reference implementation of offline/online charging models for such LES in IMS networks. Our proposal has following advantages compared to other approaches:

- It is a comprehensive framework, first ever proposed, with clearly defined functional blocks and standardized interfaces/referent points

- The first ever proposed charging model for blended services in which not only charge the service based on session ID but also based on so-called Feature Set which are set of all service processes invoked during the session.

- The OSS has multiple functions which cover various aspects of a support $\mathcal{E}$ control subsystems of an NGN networks, from charging, evaluation to policy enforcement, etc...

- Our unified test-bed has end to end functions and components that were self-designed and self-implemented by ourselves providing a federated lab environment for developing, testing and benchmarking new services and mechanisms.

The paper is divided in to five main sections. In section 2 we list the major previous works on the topic. Section 3 summarizes the most important things about architecture and interfaces in IMS framework, blended services concept and charging issues in IMS networks. Section 4 and 5 present the design and implementation of the various charging models \& policy control mechanisms for an OSS. Finally section 6 describes our comprehensive Test-bed environment as well as major implementation results.

\section{Previous Work}

To our best of knowledge, in the field of policy control in IMS, there are very few researches until recently. Amongst other, the most significant works are inside working group of 3GPP and ETSI/TISPAN, in which both standardization groups specify the mechanisms to allow P-CSCF (acts as PDP or Policy Decision Point) interacting with access routers (acts as PEP or Policy Enforcement Point) in media plane via standard protocol of COPS (Common Open Policy Services), which are defined in RFC 2748 and RFC 4261, to consult about needed resources for admission control of requesting session. For the problem of charging multimedia services in IMS, there are more works have been done in the past. The most notable works are those of Jennings and Malone [4]. In their work, they use a two-phase rating process for establishing total price of the service. In the first phase, individual prices of each media component are calculated, while in the second phase a total price of the service is calculated, depending on a combination of included components. As a further work, Kun et al. [5] propose a process for automated generation of charging schemes for multimedia services. $\mathrm{Xu}$ et al. [6] propose architecture for automating the deployment and application of charging schemes for composed ser- vices in the IMS. They use a Domain Specific Language (DSL) to develop an Accounting Logic Generator (ALG) that achieves this. This work however does not take into account the implications of real-time charging on services. Bhushan et al. [6] propose a framework for Federated Accounting which addresses the complex aspects of charging for composed services. The key issues addressed are the exchange of service usage records in a multi-domain environment considering Service Level Agreements (SLA). However, this work however is done in a non-IMS context. Ooms et el. [7] investigate an effective way to provide Authentication, Authorization and Accounting (AAA) for composed services in a multi-domain IMS using the Diameter protocol [8]. However, the focus is on the security aspects of a multi-domain environment.

And the conclusion is that there are no researches, in the past, who have tried to propose an overall architecture for a comprehensive Operation and Support Subsystem in IMS which hosts various mechanisms from charging and service quality evaluation to policy control like ours. Furthermore, our approach on charging problem is different from the above works on aspect that we focus on proposing the charging model for the blended services. Blended services are services created from combination of independent applications and services on different platform (for example, SIP \& PARLAY). This is different to an atomic service, whose implementation is self-contained and does not invoke any other service. The charging model for blended services needs to be able to charge on individual features of it rather just the service itself.

The remainder of this paper describes the main concepts in IMS as well as the details of our proposal of policy and charging control models in IMS networks. We conclude with lessons learned and future works.

\section{Policy \& Charging Control in IMS}

In this section, we introduce the basic policy control \& charging mechanisms as specified in the 3GPP IMS. Section A shows the reference architecture with all of functional blocks and interfaces/referent points while Section B, C, D \& E respectively provide an inside view of blended service concept, charging \& policy control functions in IMS networks.

\subsection{IMS Architecture and Important Interfaces}

Technically, IMS is an IP-based overlay signaling network which uses well-established Internet protocols like IETF's SIP [RFC 3261] or Diameter [RFC 3588], but requires numerous IMS-specific extensions to these protocols. IMS is access independently and hence applies to UMTS and GERAN as well as to WiMAX, WLAN or fixed networks providing end-to-end QoS guarantees to customers. For network operators, IMS offers extremely flexible online and offline charging mechanisms as well as standardized interfaces towards application servers.

The main signaling components of a typical IMS network and their interfaces are depicted in Figure 1. 


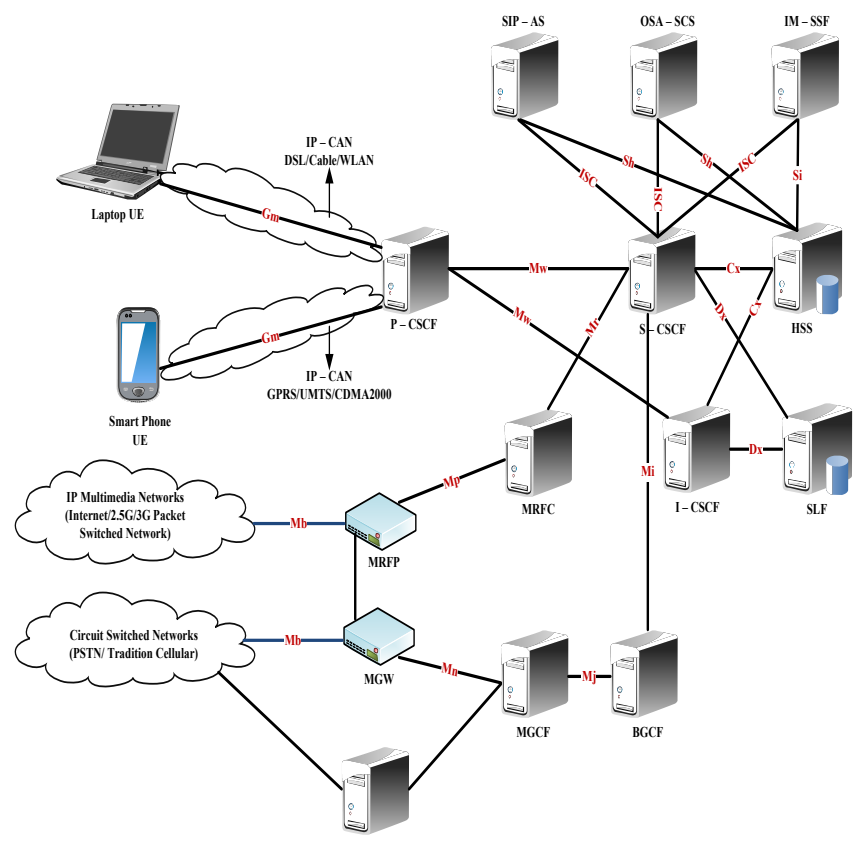

Figure 1. IMS Framework: Components \& Interfaces.

There are three main component classes in the core IMS network:

- Call-Session Control Functions (CSCF): i.e. enhanced SIP proxies. There are three basic types of CSCFs: The Proxy CSCF (P-CSCF) is the first point of contact to the User Equipment (UE) in terms of SIP signaling. It acts as inbound and outbound proxy to UE and as SIP security border gateway that protects the mobile operator's domain. The Interrogating CSCF (I-CSCF) is a SIP proxy located at the edge of an administrative domain, acting as contact for IMS signaling messages coming from other IMS domains. The I-CSCF routes SIP requests to the destination within the administrative domain and assigns a S-CSCF at registration time based on the user profile using information retrieved from the Home Subscriber Service (HSS) through the Diameter interface. The Serving CSCF(S-CSCF) is a combined SIP Registrar and SIP Proxy. It is the "brain" of the IMS with a role similar to the MSC in mobile Circuit-Switched (CS) networks. After the I-CSCF has assigned a S-CSCF to a user, the S-CSCF authenticates and registers the IMS user and stores the binding between the user's address of record and his SIP contact. Then the S-CSCF is responsible for handling/routing all signaling traffic for the respective user based on the user's profile, specifically the Initial Filter Criteria (IFC) downloaded from the HSS.

- Application Servers: (AS, see Figure 1) are SIPenabled or Non-SIP platforms and host IMS service enablers, service components or even the applications themselves.

- Database components: like the Home Subscriber Server (HSS), which corresponds to the Home Location Register (HLR) in GSM/GPRS networks. The HSS stores user profiles containing all user- related data required for IMS operation, e.g. public and private identities, subscribed services, shared secrets, Initial Filter Criteria (IFC), etc., and can be accessed by CSCFs through the Cx interface and by ASs through the Sh interface, both of them are Diameter-based.

IMS standardizes components for resource control and provision, e.g. Media Resource Function Controller (MRFC) and Media Resource Function Processor (MRFP), and components for interfacing with legacy CS voice networks like the PSTN, e.g., Breakout Gateway Control Function (BGCF), Media Gateway Control Function (MGCF), Media Gateway (MGW), and Signaling Gateway (SGW). The most instructive way to illustrate the interplay between these core components is by the following IMS registration scenario: First, the UE registers with the IMS network by sending its SIP registration request to the $\mathrm{P}-\mathrm{CSCF}$, which was assigned to the UE by the network and stays with the UE for the lifetime of the registration. The P-CSCF forwards all messages from the UE to the I-CSCF serving the user's home domain. The I-CSCF contacts the HSS, selects an S-CSCF based on the services the user has subscribed to, and forwards the registration message to this $S-$ CSCF. The latter is the service access point and service dispatcher within the IMS network, authenticating and registering the user. Finally, depending on the user profile, the S-CSCF redirects calls to one or more ASs in order to implement specific services requested by the user.

\subsection{Blended Services in IMS}

Besides those components mention at section $\mathrm{A}$ above, IMS also defined an additional functional element:

- SCIM (Service Capability Interaction Manager): This optional element manages the interaction between the different service features on various Application Servers in order to provide so-called Blended Services (ex. LES).

The purpose of the SCIM was "service capability coordination" but nothing more concrete. The result was that some contributors proposed to move up the SCIM in the service architecture, and instead of defining it as a standalone entity; they managed to get it located inside of the SIP Application Server. As a consequence, the standardization of the SCIM was limited to a few fluffy sentences in a couple of 3GPP specifications. The SCIM could then be forgotten. However, years later, as the IMS hype started, some operators started to ask for SCIMs, and some suppliers started to propose something called "SCIM". It seems that, as the IMS application layer was under-defined, the SCIM became for some the magic box that would answer all the unresolved questions. This is why we initiated a series of activities to define and implement a basic framework of such independent SCIM entity in our test-bed.

With the SCIM entity on the proposed framework, service providers can provide customers with so called blended services (other names, combined services or 


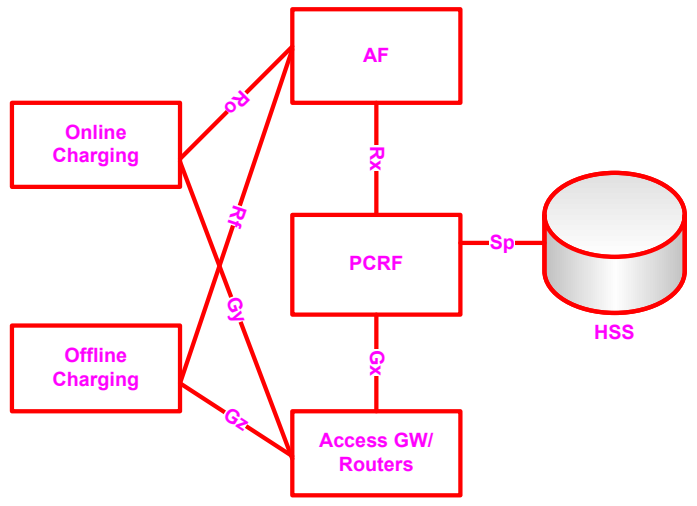

Figure 2. Policy and charging control in IMS.

orchestrated services). The blended services is a new concept emerged in IMS framework as well as in SOA architecture that provides a sophisticated signaling and service control mechanism to allow blending/orchestrating (but not bundling) multiple service processes on a single user session thus giving user very intelligent applications, like incoming call/message pop up on TV screen, parental control, mash-up (kind of LES), etc.

\subsection{Policy control in IMS}

Policy control deals with the media-level access control; the decisions made by the policy control mechanisms authorizes a user to use the media plane and assigns the QoS to be provided for that user session. The access routers/switches (figure 2) present in the network enforce the media-level policy, but these routers do not have the ability to make decisions about users, as they do not have access to the user information stored in the HSS.

A SIP server performs the task of obtaining the user information and making these decisions in this case. The SIP server informs the routers to allow or deny a certain user with the requested media resources. The node, which makes the decision, in this case, the SIP Server is called the Policy and Charging Rules (PCRF) (3GPP Release 7) and the access router is called the Policy Enforcement Point (PEP).

The PCRF receives information about the offer/answer exchanges between the terminals from the AF (either in IMS Client or in Application Server) over the $\mathrm{Rx}$ interface. If the characteristics of the session being established are acceptable to the PCRF (based on the domain policy), the PCRF authorizes the session on the access gateway using the Gx interface. If the characteristics of the session are not acceptable to the PCRF, it instructs the AF to terminate the session using the Rx interface. Obviously, in this case the PCRF does not authorize the session on the access gateway.

There are issues with this current approach to policy control and the most important issue is for informing terminals about policy decisions in the ways described above. As stated earlier, a terminal is not always informed why its session was rejected. The user of the terminal may not understand what to do to establish a new session that will be accepted. A user may also receive a successful-session-establishment indication and a session-terminated indication, one immediately after the other. Additionally, a network cannot change the policies that apply to an ongoing session. For example, if a network decides that using video is no longer acceptable, it cannot inform the terminals about this fact. Its only option is to terminate a whole session by sending BYE requests to both terminals and users will not know why the session terminated.

This issue is related to user experience and because of that we proposed a new mechanism to allow the PCRF (figure 4) receiving the information of what QoS mechanisms/parameters to enforce on the access router/switches. These QoS parameters are directly obtained from (policy control) logics in a new proposed entity, GARC, in OSS. These GARC logics are built based on user evaluation (of service experience) results so it makes the policy control perfectly fits the user's expectation on quality of service and quality of experience. More details about our solution are on section 4 .

\subsection{Online vs. Offline Charging}

Offline Charging is a charging process where charging information does not effect, in real-time the service rendered control. Offline Charging is usually applied to users who pay for their services periodically (i.e. at the end of the month). Offline charging records as specified within the IMS framework are useful in two aspects: Firstly, they provide a straightforward bridge to legacy billing mechanisms and therefore allow for a more gradual migration of current offline billing models towards IMS. Secondly, the integration of content and application partners into the more complex value chain of IMS, which is no longer restricted to the operator only, does not require to be performed in real-time and thus can benefit from the increased precision of offline data. The second use will be to support settlement in the more complex value chain which includes not just the operator but may also include content and application partners. Since such activities do not require to be performed in real-time, they can exploit the offline data sources and benefit from increased precision of data.

Online Charging is a charging process where charging information can affect, in real-time, the service rendered and therefore directly interact with the session or service. The Online Charging architecture correlates three different types of charging in real-time.

- Access Charging means charging for the usage of the access network based on a specific metric such as volume and time. It is performed using the CAP interface from the SGSN to the BCF (bearer charging function)

- Session Charging means a SIP service including a particular QoS for that session based on session duration, and is performed using the ISC interface between the SCF (session charging function) and the S-CSCF

- Event-based Charging means charging for events and contents, i.e. delivery of multimedia message, 


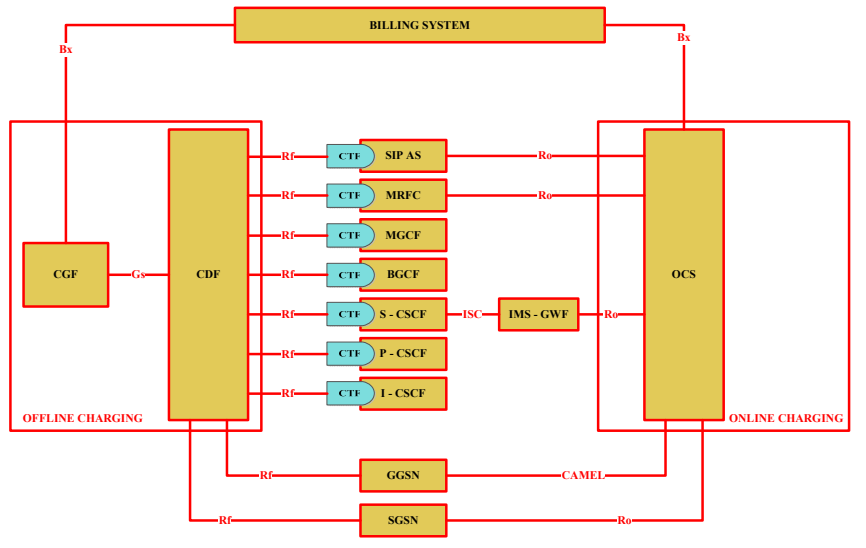

Figure 3. 3GPP IMS Charging Models.

and is performed between the application servers and the ECF (event charging function) across the Ro Interface. The ECF comprises the SCCF (subscriber content charging) and CPCF (content provider charging function)

The online charging model allows more flexibility in charging for services in particular for interacting with the user at the time of service access. This concerns a range of capabilities including indication of charge, indication of low balance, option to subscribe online, warning of end of free trial usage, warning about adult content. In addition, the online charging mechanism must provide information about the current connection for charging purposes such as roaming status, and QoS of connection. Such information and changes to it are necessary in real-time. Another important distinction to be made with regard to charging is to distinguish between charging options and charging mechanisms. This orthogonal separation contrasts technical and economical aspects of charging. Charging mechanisms, e.g.onand offline charging, are used to implement and realize charging options like pre- and postpaid charging. In other words, the distinction between pre- and postpaid charging is not equivalent to the distinction between on- and offline charging, as for instance there is no formal reason for not doing postpaid charging also in realtime. Note that in 3GPP specifications, charging options are often referred to as billing options. Figure 3 shows the model with different interfaces and charging functions proposed by 3GPP in IMS networks.

\subsection{Components Involved in Policy \& Charging Control}

S-CSCF is an IMS component responsible for handling registration processes, making routing decisions, maintaining session states and storing the service profile(s). It interacts with OCS (Online Charging System) when using SCUR (Session Charging with Unit Reservation). An example of S-CSCF in IMS Architecture would be charging for use time or duration for conference calling.

AS or Application Servers interacts with OCS for IEC (Immediate Event Charging) and ECUR (Event charging with Unit Reservation) in an IMS architecture.
An example of Application Server in IMS architecture would include charging for using a messaging service.

MRF provides media related functions such as media manipulation (for example voice stream mixing) and playing of tones and announcements. The MRF is split into Multimedia Resource Function Controller (MRFC) and Multimedia Resource Function Processor (MRFP). MRFC component may be included in the AS component. Tasks of the MRFC in an IMS charging architecture:

- Control the media stream resources in the MRFP

- Interpret information coming from an AS and S CSCF (e.g. session identifier) and control MRFP accordingly

- Generate of CDRs

- Advanced control of conferences (e.g. floor control) Tasks of the MRFP in an IMS charging architecture:

- Control of the bearer on the Mb reference point

- Provide resources to be controlled by the MRFC

- Mixing incoming media streams (e.g. for multiple parties).

- Media stream source (For Example for multimedia announcements).

- Media stream processing (Examples include audio transcoding, media analysis).

- Manage access rights to shared resources in a conferencing environment (For example floor control).

Note: The Mp reference point allows an MRFC to control media stream resources provided by an MRFP.

P-CSCF interfaces with PCRF (Policy Control Resource Function) over Rx interface. The Rx reference point enables transport of information (For example dynamic media stream information) from the application function (AF)/P-CSCF to the PCRF. An example of this would be filtered information to identify an IMS session and its connection parameters (e.g. end points, media description). This component helps with charging for a service flow (flow based charging) using an IMS service (examples include browsing, FTP etc).

\section{System Design}

To be able to provide efficient policy and charging control in a multi-platform environment the requirements of the different players need to be considered. Other requirements will arise that relate to the technical aspects of the system.

- User Requirements: As mentioned above, the users on IMS networks require to be provided with composite (or blended) services without awareness of multi-platform environment. However, Policy \& Charging control function need to have relevant mechanisms to allow the users aware of all policies \& charges enforced/incurred for all the services available to him or her. The user also requires that the network operator adheres to the SLAs and delivers services with a minimum acceptable QoS.

- Network Operator Requirements: To Operator, the important thing is that they have to minimize the loss of revenue and that they possess important 
resource usage information as related to each user to facilitate billing.

- Service Provider Requirements: Service and content providers need transparency in the revenue apportioning process. However, the most important factor is that the service provider is able to maximize revenue come from advanced features (like the blending) of delivered services

- Other Requirements: To facilitate prepaid billing to users online charging must be implemented for all services. Blended services with differentiated service providers require accurate and fair allocation of revenue. Charging for services also needs to occur in a best-charged manner for the service type.

Thus an architecture that caters for all these requirements is proposed; an implementation will be carried out to enable proof of concept and performance tests. Furthermore, our design provides the flexibility in the mode of charging that needed on the multimedia environment line IMS. It allows charging (resource usage or service consuming) based on: Session duration; Quality of service; extra features used; Flat charge; Data volume; and Event.

\subsection{Proposed Policy \& Charging Control Architecture}

Figure 4 depicts our newly proposed architecture for the Operation and Support Subsystem (OSS) in IMS networks. Two of major functions of this OSS are Charging and Policy Control which are more and more important in the next generation communication network environment nowadays. The architecture contains four major blocks which represent different entities in the value chain of multimedia communication service providers. The first block is IMS Client for end user to use and evaluate the various kinds of intelligent services; The second one is the IMS Core which belongs to network operators; The third block contains Application Servers for providing various types of services from traditional one of Voice to advanced services like IPTV, VAS, Location Enabled Services, etc.; finally the newly created block called Policy Control and Charging Subsystem and this is the focus of our paper. We propose a comprehensive framework for this OSS with two protocol stacks. OF Control is for communicating with Openflow controller (POX), the other is JDiameterPeer for communicating with application platform. On top of the Diameter stack are three main functional services, the GARC (Generic Adaptive Resource Control) hosts the logics of different policies for enforcing QoS mechanisms on the OF switches/routers based on collected user's perception of service quality (QoE values). The OCS and CDF are two blocks implementing the charging function for services providing to end users. Furthermore, this OSS subsystem interfaces with other components/subsystems via standard interfaces, mostly Diameter one.

In regard to the charging aspect, our design proposes models for both offline and online charging and this paper presents the charging modules for a typical blended

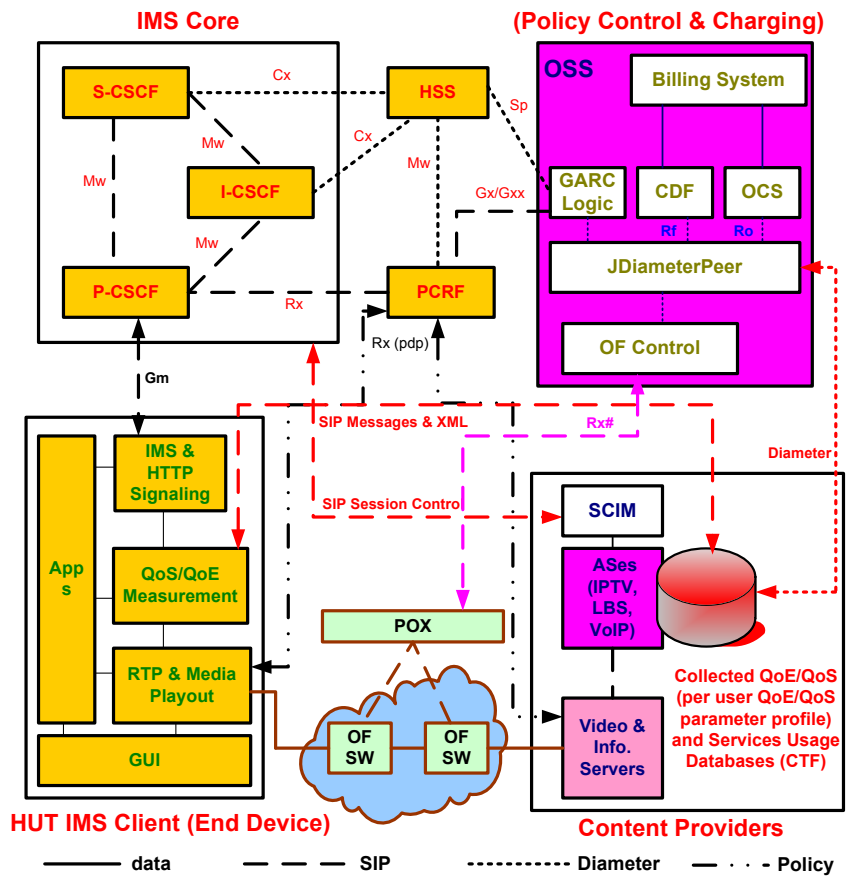

Figure 4. The overall proposed OSS architecture.

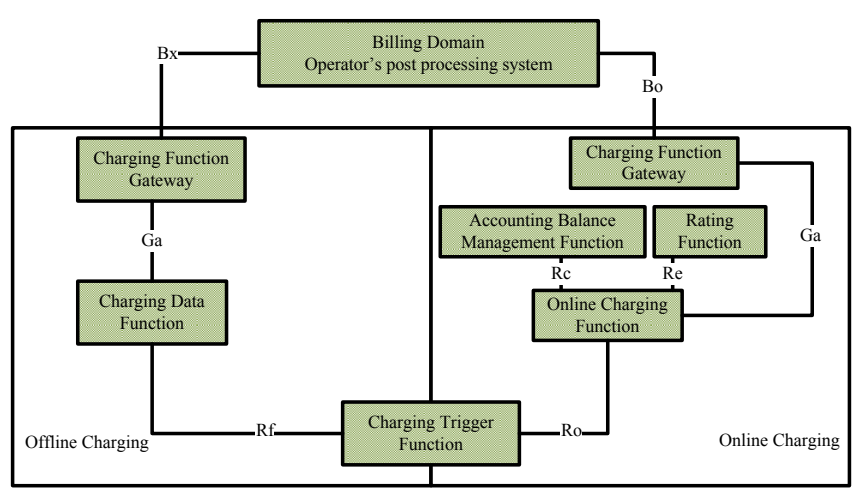

Figure 5. The detailed charging functions.

service called LES which does blending between SIP sessions and non-SIP (Parlay-X) sessions to provide users with advanced mash-up applications. LES server here acts as the CTF. Other systems and functions were also developed by our team including OCS, CDF and BS (Billing System).

Figure 5 shows the more detailed functional design of the architecture in which the system should be able to do both offline and online charging. Offline charging contains three main functional blocks: (i) the charging trigger function or CTF represents the resource and/or services that need to be charged; (ii) the charging data function or CDF which retrieves the charging information from CTF via Diameter Rf interface and then produce the CDRs (Call Details Record) from collected information and ultimately sends it to billing systems via the charging gateway function (CGF). Online charging process includes two main function blocks: CTF and OCS (Online Charging System). The OCS is combination of three sub-functions, inclusive of online charging function $(\mathrm{OCF})$, rating function (RF) and accounting balance management function (ABMF). The Diameter 
Table I

QoS PARAMETERS

\begin{tabular}{|c|l|c|c|c|c|c|}
\hline User & Content & Delay & Jitter & Bitrate & PLR & MOS \\
\hline alice 1 & Action & 0 & 0 & 230006 & 0 & 4 \\
\hline bob & talkshow & 0 & 0 & 732177 & 0 & 5 \\
\hline bob & Action & 208 & 207 & 62977 & 0 & 2 \\
\hline
\end{tabular}

Ro interface between CTF and OCF allows doing both event-based charging and session-based charging. On our design we extended the Ro interface, by adding more AVP pairs, to allow CTF to make real-time connection to OCS to check the balance of the user's account and user's usage of network resources. All of these tasks need to be done in real time for assurance of user's right as well as providing the right management of network resources. The second extension is about the information CTF transfers to OCS/CDF to allow not only charging on session ID but also the feature sets of the respective blended session.

In the aspect of policy control, our idea is how to dynamically map QoE of IPTV sessions to network-centric QoS parameters and provision the corresponding network resources efficiently so that user's service perception is satisfied while network resources are optimized based on current network conditions. The IMS Client with the QoE engine was prototyped in our test-bed will learn its user in different profiles (e.g talk show or action movie etc..). It then predicts the user's satisfaction level with the corresponding QoS parameters at the same monitoring time, periodically during the course of an on-going session. The Application Server was developed for IPTV value added services based on Sailfin platform. The AS includes QoE/QoS module that collects Opinion Score from client terminals including corresponding QoS parameters (e.g. Packet loss rate, delay, jitter) into the Table I:

The media layer is for transportation of media traffic in unicast, multicast and broadcast modes. The core transportation network is built with virtualized Openflow switches and its technology. The GARC component is also integrated to the core network. Controller Switch Interface uses the specified OpenFlow Protocol in version 1.1 [9], providing OpenFlow controller interfaces for performing Open Flow routing. Based on the collected database of each user with different service categories, the GARC logic establishes a mapping function between QoE and QoS by using the linear regression method:

$$
M o S=\alpha B r+\beta J t+\gamma P l r+\varepsilon
$$

- where: coefficients $\alpha, \beta, \gamma$, and $\varepsilon$ are calculated particularly for each case. $\mathrm{Br}$ is bit rate, $J t$ is jitter, $\mathrm{Plr}$ is packet loss rate

Based on the established mapping function with different QoS profiles that translates user-centric QoE requirements to corresponding network-centric QoS parameters, QoS modification decisions can be: changing the network configuration to get better transmission delay or packet loss rate within the transport network. The policy is then enforced on the Openflow protocol.
Table II

AVP Values for Offline Module

\begin{tabular}{|c|c|}
\hline Attributes & AVP Value \\
\hline Session Identifier & ID to identify the session \\
Feature Set & Friend_list \\
Origin Host & Ibs.ims.hut.vn \\
Origin Realm & ims.hut.vn \\
Destination Realm & ims.hut.vn \\
Accounting Request Type & START \\
Accounting Request Number & Unique request number \\
Destination Host & cdf.ims.hut.vn \\
\hline
\end{tabular}

Table III

AVP Values for Online Module

\begin{tabular}{|c|c|}
\hline Attributes & AVP Value \\
\hline Session Identifier & ID to identify the session \\
Feature Set & Friend_list \\
Origin Host & lbs.ims.hut.vn \\
Origin Realm & ims.hut.vn \\
Destination Realm & ims.hut.vn \\
Credit Control Request Type & INITIAL_REQUEST \\
Credit Control Request Number & Unique request number \\
Destination Host & cdf.ims.hut.vn \\
\hline
\end{tabular}

\subsection{Charging Process}

To illustrate the charging process for blended services, this section presents it in details with 6 steps as follows:

Step 1: IMS Client will send a SIP INVITE Message to the LES server via IMS Core for requesting the service. LES server will analyze the message and collect the relevant information for both service processing and charging purposes

Step 2: LES acting as CTF will then send the message to the offline and online charging modules with collected information. The request send to offline module has this format:

$$
\begin{aligned}
c d f= & R f \_A C R(\text { session_id,feature_set, origin_host, } \\
& \text { origin_realm, destination_realm, START, } \\
& \text { acr_number, destination_host })
\end{aligned}
$$

This ACR message contains following Diameter AVP values (presented in Table II):

Similarly the request send to online module has following format:

$$
\begin{aligned}
o c f= & R o \_A C R(\text { session_id, origing_host, } \\
& \text { origin_realm, destination_realm, } \\
& \text { INITIAL_REQUEST,Cc_number, destination_host })
\end{aligned}
$$

and following Diameter AVP Values included on this message (presented in Table III):

In both cases, the LES/CTF will wait for the response from the respective charging modules before delivering the service to the IMS Client. For the offline case, the response from $C D F$ includes a charging record with the session ID and for online case, besides sending back the response to LES, the OCF also need to send a request to check the balance of the user with the session ID.

Step 3: After receiving the responses from $\mathrm{CDF} / \mathrm{OCF}$ with valid codes, the LES feedbacks to IMS Client with SIP 200 OK.

Step 4: IMS Client and LES are in the service delivering phase together. 


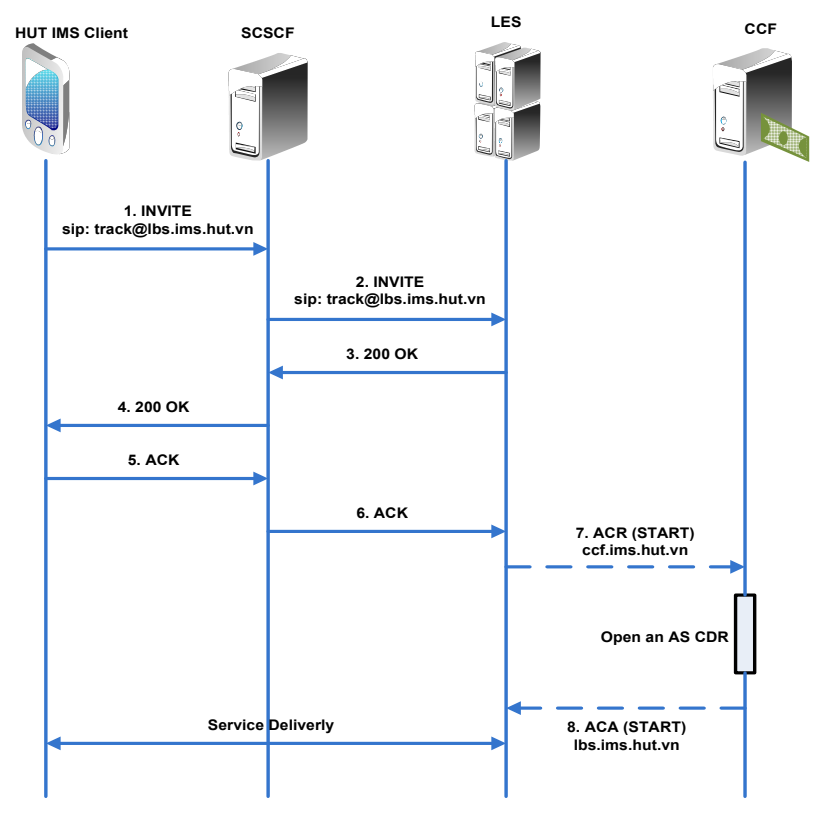

Figure 6. Offline charging procedure for LES.

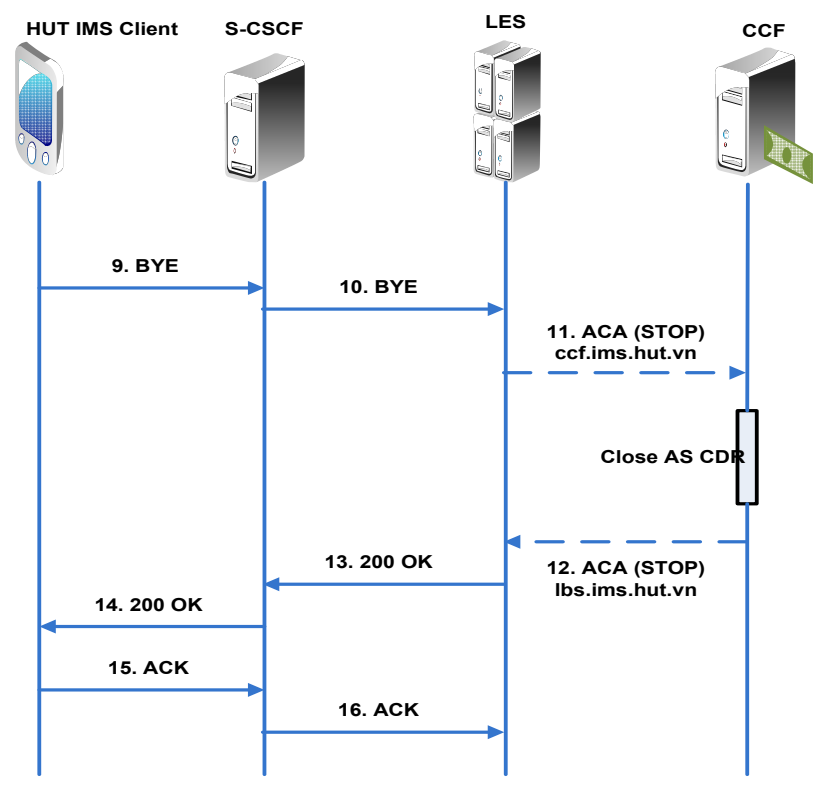

Figure 7. Offline charging procedure for LES (cont.).

Step 5: The added Timer on LES/CTF will count the time of service consuming and periodically send the message to OCF to keep the service delivery running and also to inform the OCF that the session is running smoothly without any errors.

Step 6: After IMS Client terminates the session, LES will send message to $\mathrm{CDF} / \mathrm{OCF}$ for terminating the charging duration and also wrap-up the charging session.

Those things mentioned above are call flows for those two charging scenarios that bring more details of the process to reader. Figure 6 and figure 7 show the procedure of exchange messages for offline charging of LES service while figure 8 and figure 9 illustrate the more complicated procedure for online charging of LES service.

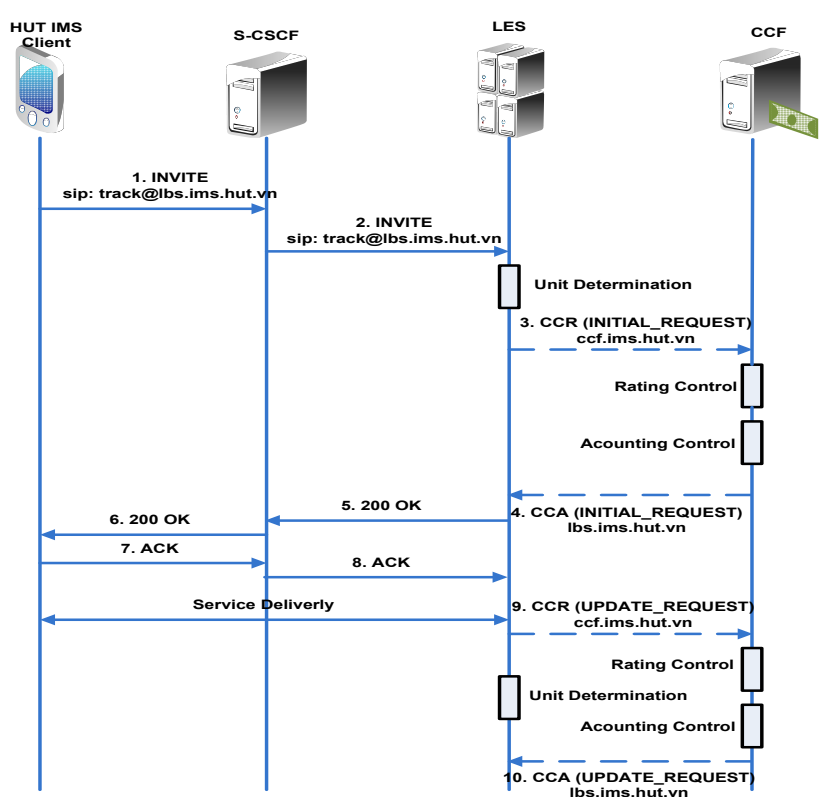

Figure 8. Offline charging procedure for LES.

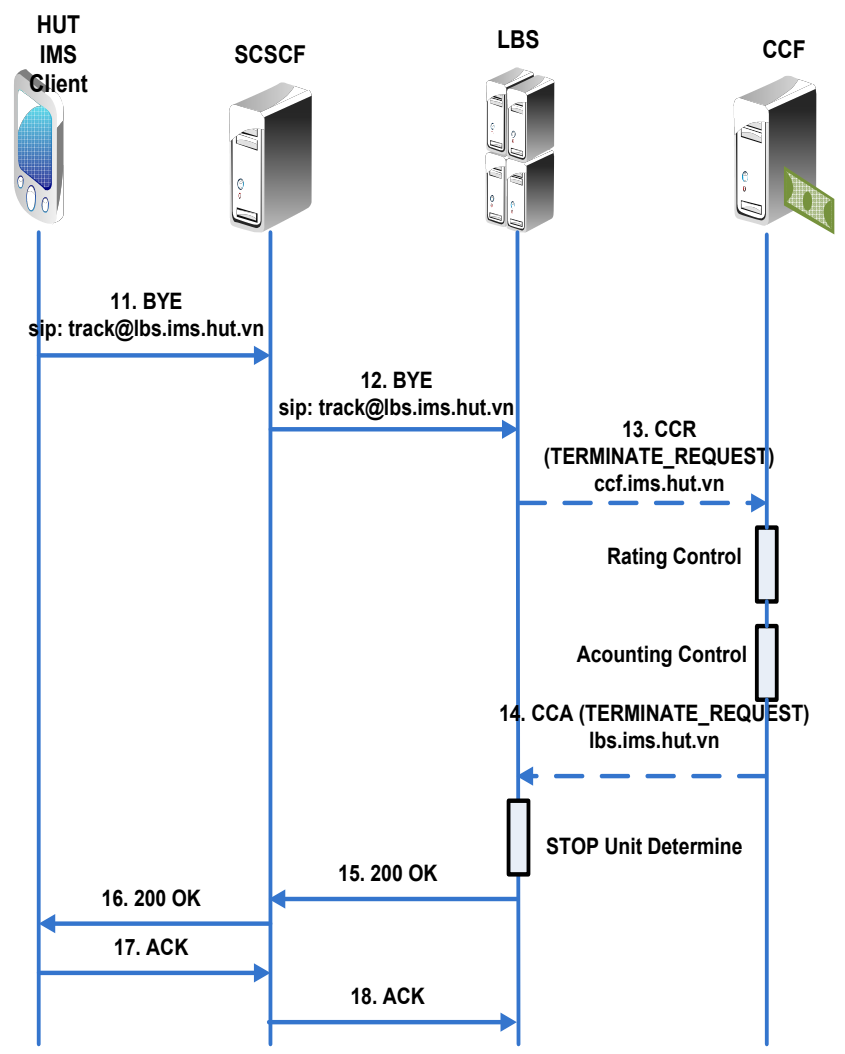

Figure 9. Online charging procedure for LES (cont.).

The interfaces among the components in our architecture including GARC and the QoE AS are defined as follows:

- OpenFlow controller: Interface between GARC and OpenFlow capable controller e.g. POX [9]for exchanging OpenFlow specific messages (JSON via TCP) for transmitting network control and management information, network monitoring data and statistics. 


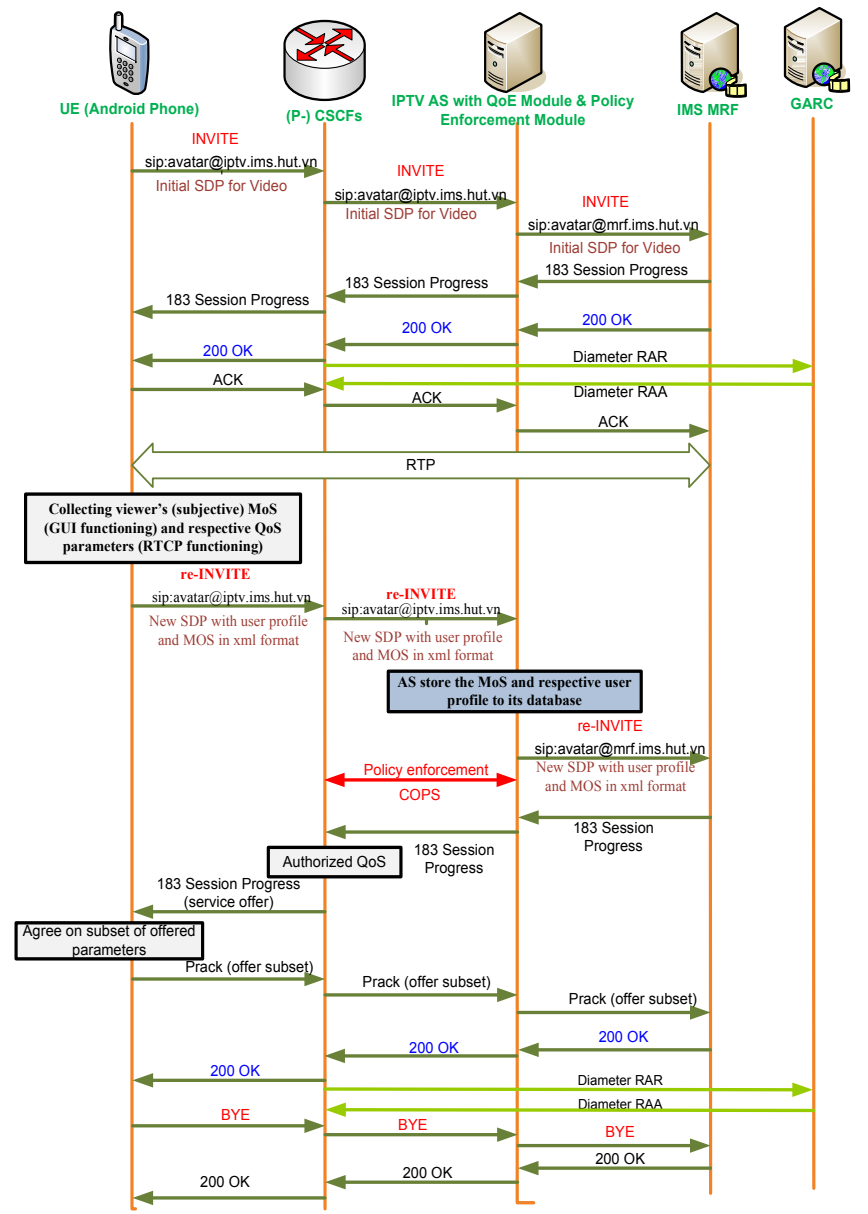

Figure 10. Call flow of QoS-QoE mapping based policy control mechanism.

- OF switch: For controlling flow-to-queue mapping over switch specific DPCTL messages, which in turn enables QoS differentiation between individual flows

- P-CSCF of the IMS core: Diameter Gx for QoS provisioning in combination with the Diameter Gxx reference point for bearer-binding and eventreporting-functions.

- HSS/HLR or user profile database: Diameter extended $\mathrm{Sp}$ interface for querying static or dynamic user profile information.

- AS: Diameter extended Rx interface for signaling application layer QoS requirements from networkaware application towards GARC.

Interface OF controller - OF switch: Controller Switch Interface using the Open-Network-Foundation (ONF) specified OpenFlow Protocol in version 1.1 [9] providing standard OpenFlow controller interfaces for performing OpenFlow conform routing.

Interface IMS client - AS: SIP and XML. XML for exchanging collected data of $\mathrm{QoE}$ and corresponding QoS spectrum from the client to the AS.

Figure 10 depicts a SIP based session setup and QoS parameter negotiation between UE and IMS MRF resulting in a multimedia session. In the beginning, the UE initiates a multimedia session using a SIP INVITE indicating the terminal QoS parameter in terms of

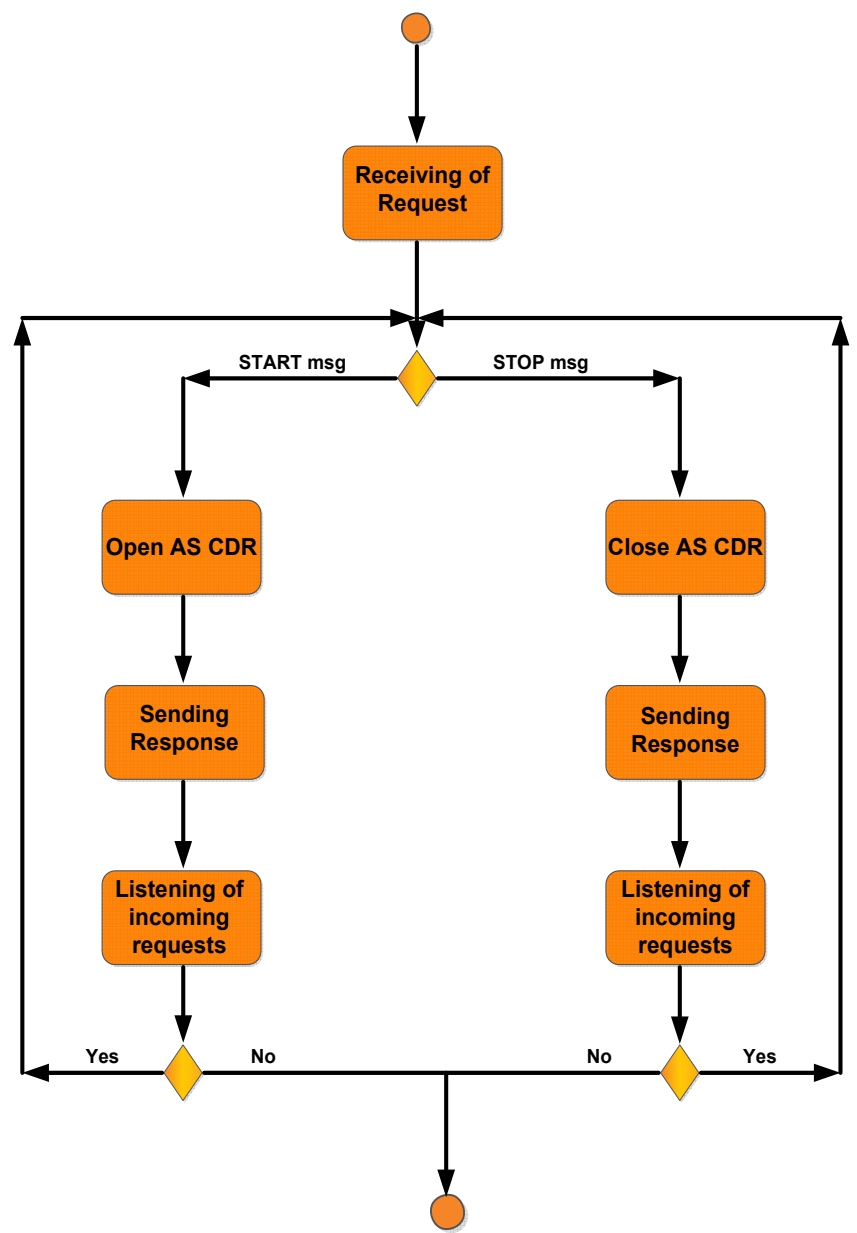

Figure 11. Implementation of online charging model.

supported codecs, bit rate, etc. The QoS parameters are transported in the body of the SIP message as SessionDescription-Protocol (SDP) including a set of supported QoS parameter for selection. The SIP INVITE signaled to the P-CSCF and is forwarded on to application server IMS MRF using S-CSCF for application server selection.

\section{SYstem IMPLEMENTATION}

From the design above, we had implemented the proposed concepts and models for both of charging and policy control as details below.

\subsection{Charging Models Implementation}

Figure 11 shows the main activities of OCF. When Listener module receives the request through $\mathrm{R} 0$ interface, it will analyze to know which kind of incoming request is. It could be one of three cases: (i) if it is the Trigger request, the $\mathrm{OCF}$ will start the charging process and response back the charging-start confirmation; (ii) if it is the Updating request, in that case the charging process continue and OCF send back the response that charging is running as usual; (iii) it is the Termination request, then the OCF will stop the charging process and send back the termination confirmation. Figure 12 is the UML use cases of this online charging procedure. 


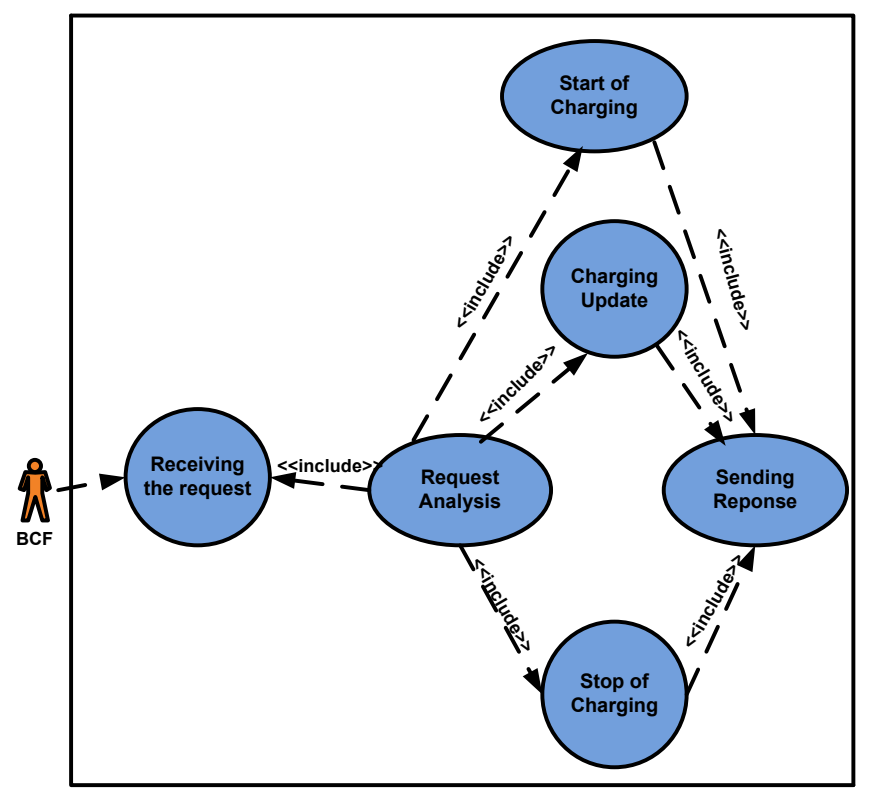

Figure 12. The convergence of the parameter vector.

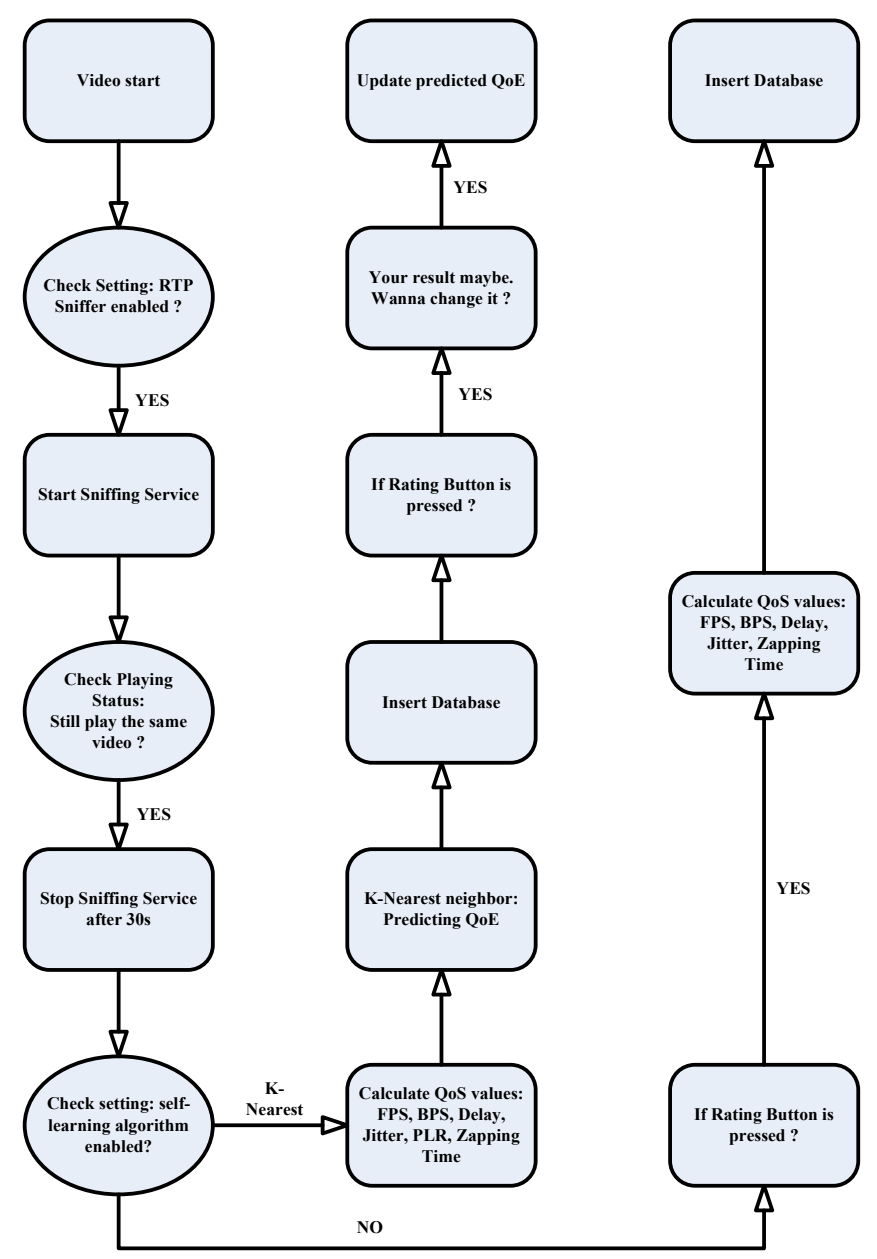

Figure 13. The convergence of the parameter vector.

\subsection{Implementation of Mechanism for Policy Control}

The proposed interfaces and functional blocks of the architecture on figure 4 was implemented with specific software modules of:

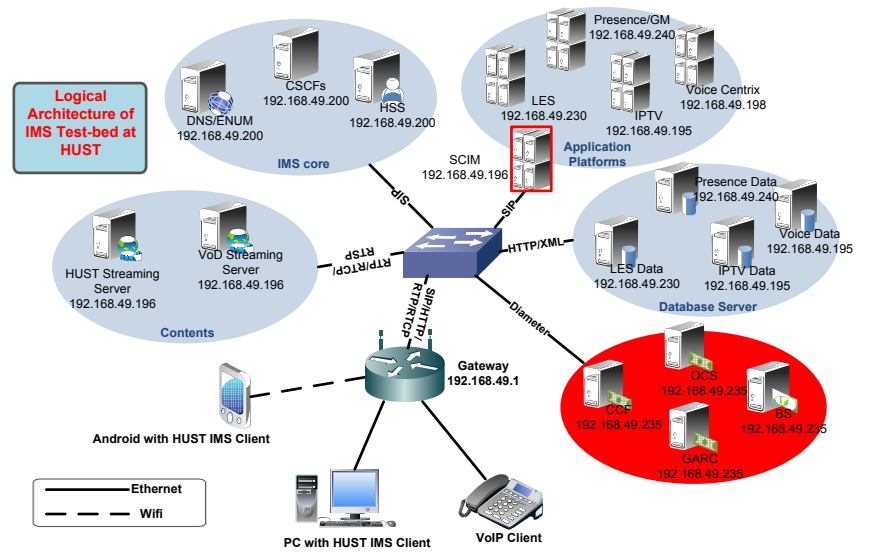

Figure 14. HUST's Unified IMS Test-bed.

- QoE Engine that allow (IPTV) viewers to subjectively evaluate the quality of the movie from his/her HUT IMS Client GUI and IPTV AS will collect these evaluation results to its database (figure 13)

- The ASes also periodically measure QoS parameters (jitter, delay, packet loss) during the session and at the AS the specicial software module will do the mapping between collected viewer's QoE Scores to this QoS parameters set [10].

- The GARC software in OSS will through the Diameter interfaces read this mapping functions/results and implement the respective logics to tell POX to enforce specific QoS models on the OF switches to guarantee/enhance the user's QoE.

\section{Testbed And Protyping}

In scope of our national researching program named "Development of the new service framework for 3G/4G and broadband Internet using IMS technology", we had designed and built a unified test-bed platform (figure 14). All components of the Test-bed except the IMS Core (CSCFs servers) have been designed and developed by our research team.

Figure 16 shows the CCR message (Command code $=$ 272) sent from LES server to the OCS for requesting the charging function and figure 17 presents the GUI OCS system that an online charging procedure is started after configuration of charging account for user and service.

We also tested a scenario of four different users streaming multimedia content over UDP. Figure 18 shows how 4 the-same-initial-QCI-level Service-DataFlows (SDF) can be separated into 4 different QCI levels with particular QoS levels in the OpenFlow network having minimum guaranteed and maximum bandwidth limitations. The modification happened from 20 second to 80 second and reassigned from 80 second to 140 second. Besides, Table IV describes how the GARC logic component defined separate QoE-QoS functions for each user with each different content category as generalized in equation (1). 


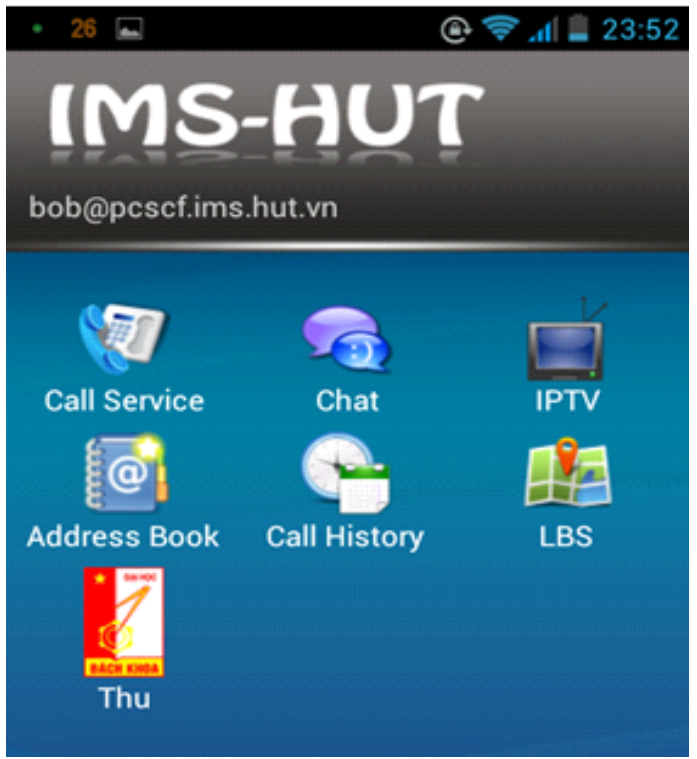

Figure 15. GUI of HUT IMS Client on Android with various categories of multimedia and location enabled services.

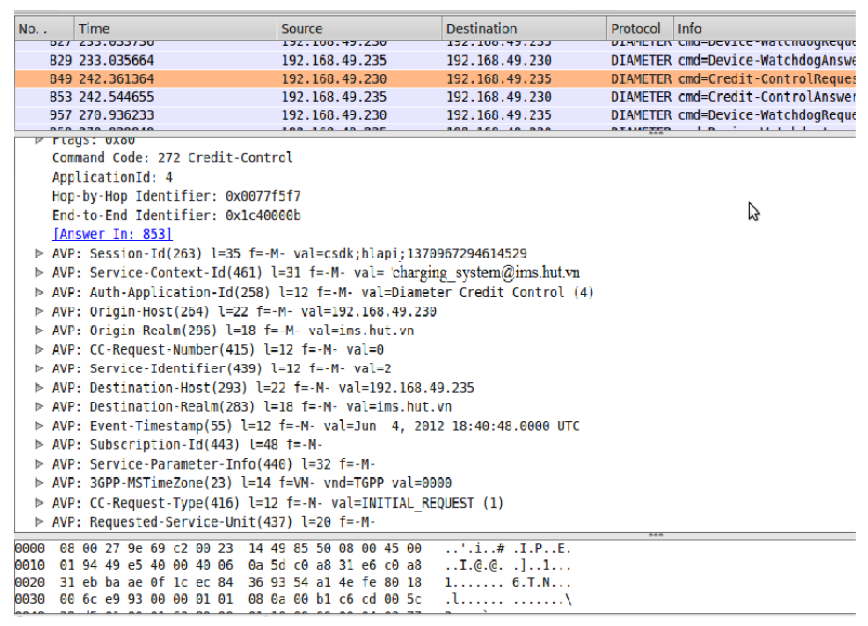

Figure 16. The CCR of online charging procedure.

Table IV

QoS-QoE MAPPINg EFFicient

\begin{tabular}{|c|c|c|c|c|c|}
\hline User & Content & $\alpha$ & $\beta$ & $\gamma$ & $\varepsilon$ \\
\hline alice 1 & Action & 0.003768 & -0.00397 & -0.430450 & 3.03429 \\
\hline bob & talkshow & 0.004324 & -0.00283 & -0.254657 & 2.78656 \\
\hline bob & Action & 0.003376 & -0.00439 & -0.714890 & 3.54069 \\
\hline
\end{tabular}

\section{Conclude And Future Work}

This paper presents our proposed ideas and models of a comprehensive architecture of an Operation and Support Subsystem for IMS networks. Our research has pointed out the necessity of completing the architecture and implementation of the various processes (charging, gathering media stream information, mapping QoSQoE, enforcing policy, etc...) in order to allow the IMS technology to be deployed in real network environment. The proposed architecture makes use of the advanced concept of service combination with usage of our own SCIM component. These results pave firm initial steps on the way toward completing the whole OSS sub-system for 3GPP's IMS architecture in NGNs.

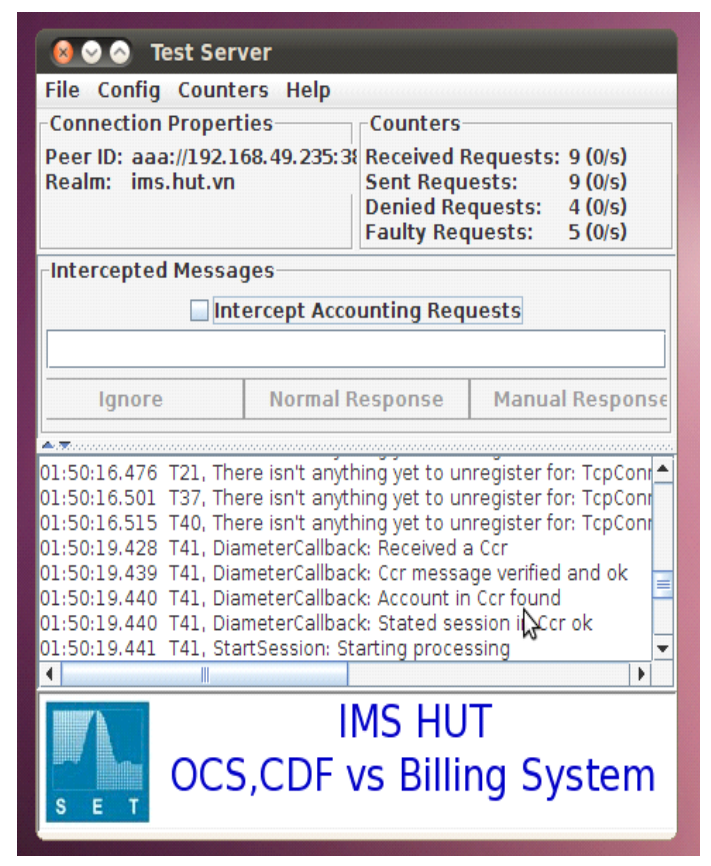

Figure 17. The online charging procedure started.

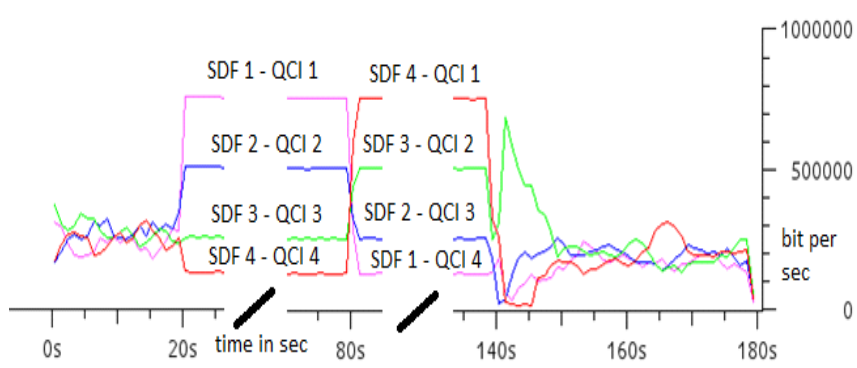

Figure 18. Policy control scenario validation measurement.

The research is in fact our on-going work; we currently work on the charging model and software for other popular services platform SOA/Web2.0 and enhancing the policy control mechanisms.

\section{ACKNOWLEDGMENT}

The author would like to acknowledge the National Foundation for Science and Technology Development (NAFOSTED) of Vietnam for funding this work.

\section{REFERENCES}

[1] R. R. Kodali. (2005, Jun) An introduction to SOA.

[2] TS 23.328, IP Multimedia Subsystem, Std., Rev. Release 6.

[3] G. A., C. N., and B. E., "Scim (service capability interaction manager) implementation issues in ims service architecture," IEEE International Conference on Communications, vol. 4, pp. 1748-1753, Jun 2006.

[4] J. B. and M. P., "Flexible charging for multi provider composed services using a federated, two-phase rating process," in IEEE/IFIP Network Operations E Management Symposium, 2006.

[5] L. Xu and B. Jennings, "Automating the generation, deployment and application of charging schemes for composed ims services," in 10th IFIP/IEEE International Symposium on Integrated Network Management, May 2007. 
[6] B. Bhushan, M. Tschichhols, E. L. W., and Connelly, "Federated accounting: Service charging and billing in a business to business environment," in IFIP/IEEE International Symposium on Integrated Network Management (IM 2001), 2001, pp. 107-121.

[7] W. Ooms, "Providing aaa with the diameter protocol for multi-domain interacting services," Submitted, University of Twente, Jun 2007.

[8] G. R., S. R., and D. D., “Centralized service capability interaction manager (scim) architecture to support dynamic-blended services in ims network," in 2nd International Conference on Internet Multimedia Services Architecture and Applications (IMSAA 2008), Dec. 2008, pp. $1-5$.

[9] Openflow project and protocol. [Online]. Available: http:/ / www.openflow.org/

[10] T. T. Huong, N. T. Hung, and N. H. Thanh, "Service provisioning with quality-of-experience support in imsbased iptv," in The fourth international conference on ubiquitous and future networks (ICUFN), Phuket, Thailand, Jul. 2013.

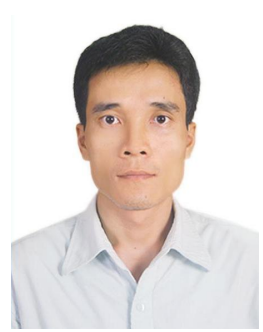

Nguyen Tai Hung $(\mathrm{PhD})$ is lecturer in the school of electronic \& telecommunications at the Hanoi University of Science and Technology in Vietnam. He got his Master and PhD degrees, both in communication engineering, from Hanoi University of Technology in 2001 and 2007, respectively. He is the (co)author of around ten of paper/articles in the fields of Internet engineering and next generation networks. He spent a half of year with Fraunhofer Institute of Fokus in Berlin, Germany for conducting the research project of service development for 3G/NGN networks. Based on his more than 10 years of experience in the teaching complex IT and telecommunication technologies to different courses in an easy to digest way, Dr. Nguyen Tai Hung gains an extensive knowhow of next generation networks and services.

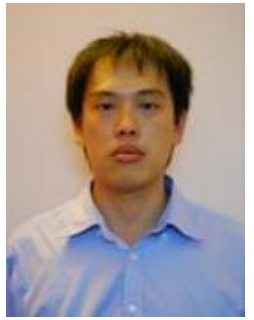

Nguyen Huu Thanh received BEng and MSc degrees in Electrical Engineering from Hanoi Uni-versity of Science and Technology (Vietnam) in 1993 and 1995, respectively. He was awarded a $\mathrm{PhD}$ degree with summa cum laude in Computer Science from the University of Federal Armed Forces Munich (Germany) in 2002. In 2002 he joined Fraunhofer FOKUS in Berlin and worked on the areas of QoS-guarantees for multimedia in overlay networks. From 2004 until now he has been working for Hanoi University of Science and Technology, Vietnam. His research focuses on mobility, QoS and resource management of wireless broadband networks, new service platforms for future networks and the Future Internet.

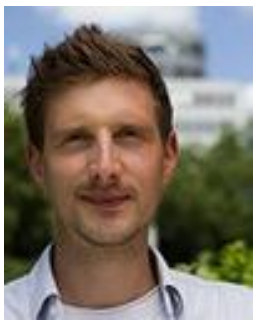

Julius Mueller studied computer science at the Freie Universität Berlin and wrote his diploma thesis about NGN/IMS and Peer to Peer (P2P) system integration. In 2009 he joined the chair "Architektur der Vermittlungsknoten $(\mathrm{AV}) "$ at the electrical engineering and computer sciences faculty within the Technische Universität Berlin as PhD researcher and lecturer. His scientific work and $\mathrm{PhD}$ focuses on the evolution of NGNs towards the Future Internet (FI). Particularly he is investigating Evolved Packet Core (EPC), Software-DefinedNetworks (SDN), Policy Control and Flow Based Charging (PCC) and Cross-Layer Composition within NGNs and the FI. Since February 2013 he joined the group NGNI in Fraunhofer FOKUS on supporting the research and developments in the scope of the OpenEPC projects. $\mathrm{He}$ is leading the Software-Defined-Networking developments of FOKUS and AV and coordinates the application of SDN concepts in telecommunication networks. 